\title{
Development of a Global Toroidal Gyrokinetic Vlasov Code with New Real Space Field Solver*)
}

\author{
Kevin OBREJAN, Kenji IMADERA, Ji-Quan LI and Yasuaki KISHIMOTO \\ Graduate School of Energy Science, Kyoto University, Uji, Kyoto 611-0011, Japan
}

(Received 26 November 2014 / Accepted 9 February 2015)

\begin{abstract}
This work introduces a new full-f toroidal gyrokinetic (GK) Vlasov simulation code that uses a real space field solver. This solver enables us to compute the gyro-averaging operators in real space to allow proper treatment of finite Larmor radius (FLR) effects without requiring any particular hypothesis and in any magnetic field configuration (X-point, D-shaped etc). The code was well verified through benchmark tests such as toroidal Ion Temperature Gradient (ITG) instability and collisionless damping of zonal flow.
\end{abstract}

(c) 2015 The Japan Society of Plasma Science and Nuclear Fusion Research

Keywords: Gyrokinetics, Vlasov simulation, field solver, finite Larmor radius effect

DOI: $10.1585 / \mathrm{pfr} .10 .3403042$

\section{Introduction}

Five-dimensional gyrokinetic (GK) global simulation is considered to be an essential tool to understand microscale instability and associated turbulent transport phenomena including the profile stiffness/resilience and transport barrier formation. While many GK codes already exist, most of them rely on particular configurations or constraining hypotheses to simplify the equation systems.

We have developped a new global full-f toroidal GK Vlasov code, GKNET (GyroKinetic Numerical Experiment of Tokamak), based on a rigorous Hamiltonian derivation of the Vlasov equation and a field solver in which the gyro-averaged operators are computed in real space [1] without any simplification (Pade approximation, constant temperature profile etc). An important property of this method is that it can be used with all sort of configurations and is independent of the coordinate system (polar or Cartesian coordinates in the poloidal plane).

This paper is organised as follows. The theoretical model used as well as the Vlasov part of the solver is described in section 2. Section 3 describes extensively the general idea of the computation of the gyro-averaging operators in real space. The implementation and numerical aspects are presented in section 4. Finally the simulations used to benchmark the code and numerical results are given in the section 5 .

\section{Physical Model}

The gyrokinetic equations are derived in the 5D gyrocentre coordinate system $\left(\boldsymbol{R}, \mu, v_{/ /}\right)$where $\boldsymbol{R}$ denotes the position of the guiding center, $\mu=m_{\mathrm{s}} v_{\perp}^{2} /(2 B)$ the magnetic momentum, $v_{/}$the parallel velocity and all variables

author'se-mail: obrejan.kevin.82u@st.kyoto-u.ac.jp

*) This article is based on the presentation at the 24th International Toki Conference (ITC24). are averaged over the gyro-angle $\alpha$. The code was originally developed using polar coordinates in the poloidal plane $\boldsymbol{R}=(r, \theta, \phi)$ for initial testings, but it was later changed to Cartesian coordinates $\boldsymbol{R}=(R, Z, \phi)$ in order to deal with non circular domains, avoid difficulties that arise near the magnetic axis (high coefficients and prohibitively strict CFL conditions). The gyrokinetic Vlasov equation which describes the evolution of the guiding center distribution $f_{\mathrm{s}}$ of the specie concerned is derived using Hamiltonian mechanics [2] as:

$$
\frac{\partial f_{\mathrm{s}}}{\partial t}+\dot{\boldsymbol{R}} \cdot \frac{\partial f_{\mathrm{s}}}{\partial \boldsymbol{R}}+\dot{v}_{/ /} \frac{\partial f_{\mathrm{s}}}{\partial v_{\|}}=0
$$

where the two time derivatives $\dot{\boldsymbol{R}}$ and $\dot{v}_{/ /}$are defined as:

$$
\begin{aligned}
\dot{\boldsymbol{R}}= & v_{/ /} \boldsymbol{b}+\frac{1}{m_{\mathrm{s}} B_{\|}^{*}} \boldsymbol{b} \\
& \times\left(e_{\mathrm{s}} \boldsymbol{\nabla}\langle\Phi\rangle_{x}+\frac{E^{\prime}}{B} \boldsymbol{\nabla} B+\frac{v_{\|}^{2}}{B}(\boldsymbol{\nabla} \times \boldsymbol{B}) \times \boldsymbol{b}\right), \\
\dot{v}_{/}= & \frac{-1}{m_{\mathrm{s}} B_{\|}^{*}} \boldsymbol{B}^{*} \cdot\left(e_{\mathrm{s}} \boldsymbol{\nabla}\langle\Phi\rangle_{x}+\mu \boldsymbol{\nabla} B\right),
\end{aligned}
$$

with $E^{\prime}=m_{\mathrm{s}} v_{\|}^{2}+\mu B, m_{\mathrm{s}}$ and $e_{\mathrm{s}}$ the mass and charge of the considered specie and where $\boldsymbol{B}=\boldsymbol{B} \boldsymbol{b}$ denotes the magnetic field of direction given by the unit vector $\boldsymbol{b}, B_{\|}^{*}=\boldsymbol{B}^{*} \cdot \boldsymbol{b}$ is the parallel component of $\boldsymbol{B}^{*}=\boldsymbol{B}+\left(B v_{/ /} / \Omega_{\mathrm{s}}\right) \boldsymbol{\nabla} \times \boldsymbol{b}$ with $\Omega_{\mathrm{s}}=e_{\mathrm{s}} B / m_{\mathrm{s}}$. The notation $\langle\Phi\rangle_{x}$ refers to the (simple) gyro-averaging (from the particle coordinates $\boldsymbol{x}$ to gyrocenter coordinates $\boldsymbol{R})$ :

$$
\langle\Phi\rangle_{x}(\boldsymbol{R})=\frac{1}{2 \pi} \int \Phi(\boldsymbol{x}) \delta(\boldsymbol{R}+\boldsymbol{\rho}-\boldsymbol{x}) \mathrm{d} \boldsymbol{x} \mathrm{d} \alpha .
$$

The electrostatic potential $\Phi$ is given by the GK quasineutrality condition: 


$$
\begin{aligned}
& \frac{e_{\mathrm{i}}}{T_{\mathrm{i}}}\left(\Phi-\langle\langle\Phi\rangle)+\frac{e}{T_{\mathrm{e}}}(\Phi-\bar{\Phi})\right. \\
& \quad=\frac{m_{\mathrm{i}}^{2}}{n_{0}} \int B^{*}\left\langle f_{\mathrm{i}}-f_{0}\right\rangle_{\boldsymbol{R}} \mathrm{d} v_{/ /} \mathrm{d} \mu,
\end{aligned}
$$

with $f_{0}$ the equilibrium distribution $T_{\mathrm{i}}$ and $T_{\mathrm{e}}$ the ion and electron temperature, $n_{0}$ the equilibrium density and $\bar{\Phi}$ is the magnetic flux surface averaging of $\Phi$. The notation $\langle\cdot\rangle_{R}$, similar to the one seen previously, here corresponds to the simple gyro-averaging from gyro-center coordinates $\boldsymbol{R}$ to the particle coordinates $\boldsymbol{x}$ :

$$
\langle f\rangle_{\boldsymbol{R}}\left(\boldsymbol{x}, \mu, v_{/ /}\right)=\frac{1}{2 \pi} \int f \delta(\boldsymbol{R}+\boldsymbol{\rho}-\boldsymbol{x}) \mathrm{d} \boldsymbol{R} \mathrm{d} \alpha,
$$

while $\langle\langle\cdot\rangle\rangle$ corresponds to the so called double gyroaveraging:

$$
\left\langle\langle\Phi\rangle(\boldsymbol{x})=\frac{1}{2 \pi} \int\langle\Phi\rangle_{\boldsymbol{x}} f_{\mathrm{M}} \delta(\boldsymbol{R}+\boldsymbol{\rho}-\boldsymbol{x}) \mathrm{d} \boldsymbol{R} \mathrm{d} \alpha \mathrm{d} \mu,\right.
$$

with $f_{\mathrm{M}}$ a Maxwellian in $v_{\perp}$, slowly varying in $\boldsymbol{R}$ such $f_{0}$ 's dependence is of the form $f_{0}\left(\boldsymbol{R}, 0, v_{\|}\right) f_{\mathrm{M}}(\boldsymbol{R}, \mu)$ (i.e. a Gaussian distribution of variance the thermal velocity $v_{\text {th }}$ ). These two operators can be easily approximated in Fourier space in the case of Cartesian coordinates (or locally):

$$
\widehat{\langle\Phi\rangle}_{k}=\widehat{\Phi}_{k} J_{0}\left(\frac{k_{\perp} v_{\perp}}{\Omega_{\mathrm{s}}}\right)
$$

where the subscript has been dropped to include both $\langle f\rangle_{\boldsymbol{R}}$ and $\langle\Phi\rangle_{x}$ and

$$
{\widehat{\langle\Phi \Phi\rangle_{k}}}=\widehat{\Phi}_{k} \Gamma_{0}\left(\left(\frac{k_{\perp} v_{\mathrm{th}}}{\Omega_{\mathrm{s}}}\right)^{2}\right) .
$$

For circular configurations of radius $a$, the code also utilises an equilibrium function as introduced in [3], a Maxwellian distribution slightly reshaped to minimise poloidal flows. In this distribution, the small radius $r$ is replaced by the motion invariant:

$$
\bar{r}=r_{p}-\frac{q\left(r_{p}\right)}{r_{p}} \Psi(r)-\frac{m q_{p}}{e B_{0} r_{p}}\left(R v_{/ /}-R_{0} \bar{v}_{/ /}\right),
$$

with $r_{p}=a / 2$ the half radius, $\Psi(r)=-B_{0} \int_{r_{p}}^{r} \frac{r^{\prime} \mathrm{d} r^{\prime}}{q^{\prime}}$ and $\bar{v}_{/ /}=\operatorname{sign}\left(v_{/ /}\right) \sqrt{2\left(E-\mu B_{\max }\right) / m} H\left(E-\mu B_{\max }\right)$ (where $H$ is the Heavyside function).

\section{Real Space Computation of the Gyro-Averaged Terms of the GK Quasi-Neutrality Condition}

Numerically, the simple averaging of a field $\langle\Phi\rangle$ at a point $P$ is simply computed as the average of $\Phi$ over a given number of sample points $M$ uniformly distributed on a circular orbit of radius $\rho=v_{\perp} / \Omega_{\mathrm{s}}$ (the green points on the back circle in Fig. 1).

Instead of defining directly the double averaging $\langle\langle\Phi\rangle$ as defined in eq. (7), which is integrated over $\mu$, let's first

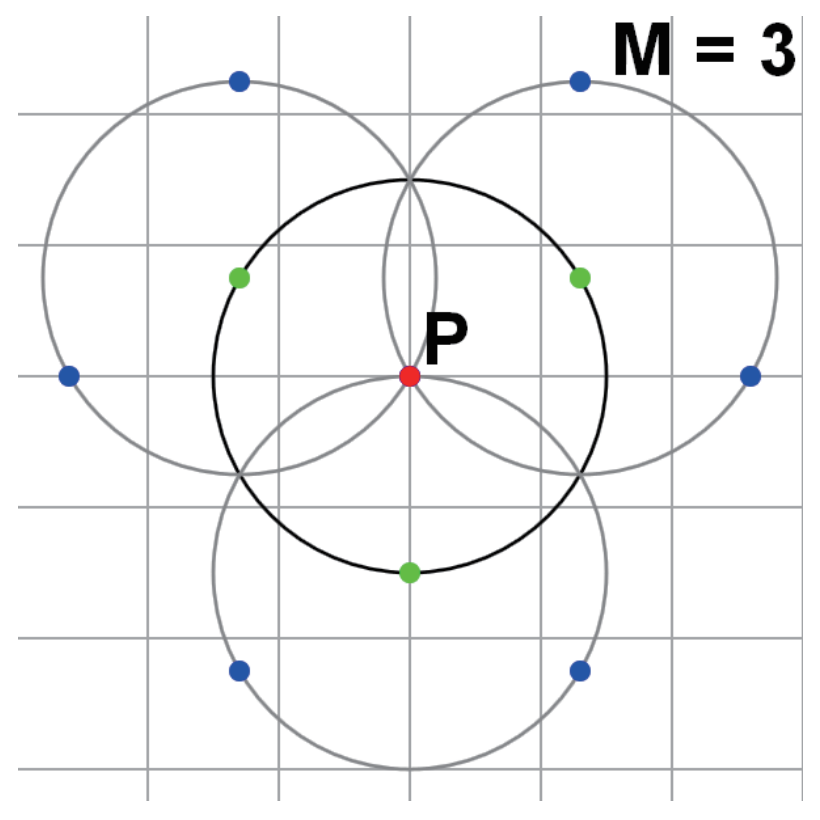

Fig. 1 Primary (black) and secondary (grey) circles used to compute the simple and double gyro-averagings in real space (blue and red points) for $C=1$ radius and $M=3$ points per circle.

consider the simpler case of the single radius double averaging $\left\langle\langle\Phi\rangle_{\rho}\right.$ (before integration). It is simply the composition of two simple averagings and can thus be performed by sampling over $M^{2}$ "secondary points" on "secondary" circular orbits centered on each "primary point" (the blue and red points on the grey circles in Fig. 1). Since the points are unlikely to be aligned with the grid, the values are interpolated. This interpolation is the only geometry dependant part of the solver and can either be done in 2D (in the poloidal plane only) or in $3 \mathrm{D}$, allowing to take into account the non perpendicularity of the magnetic field lines to the poloidal plane.

This single radius double averaging $\left\langle\langle\Phi\rangle_{\rho}\right.$ needs to be multiplied by a Maxwellian and integrated over $\mu=$ $m_{\mathrm{s}} v_{\perp}^{2} /(2 B)$ to obtain the actual averaging $\langle\langle\Phi\rangle\rangle$ as it appears in the GK quasi-neutrality condition (5). Numerically, this integration is approximated by a weighted sum of $\langle\langle\Phi\rangle\rangle_{\rho}$ for $C$ given radii:

$$
\left\langle\langle\Phi\rangle \rrbracket \simeq \sum c_{j}\langle\langle\Phi\rangle\rangle_{\alpha_{j} k_{\perp} \rho_{i}}\right.
$$

where the $2 \mathrm{C}$ coefficients $c_{j}$ and $\alpha_{j}$ are computed numerically to minimise the $L^{\infty}$ error of this approximation under.

Now that the simple and double gyro-averagings have been properly defined, we show the numerical error of these two methods. The numerical error originates from three approximations: the weighted sum explained above, the interpolation error (as the points are not aligned on the grid) and the discrete averaging over particles' orbits. While it is clear that the first two are controlled respectively by taking more circles and higher order interpolations, the last one requires a few calculations to clarify to 


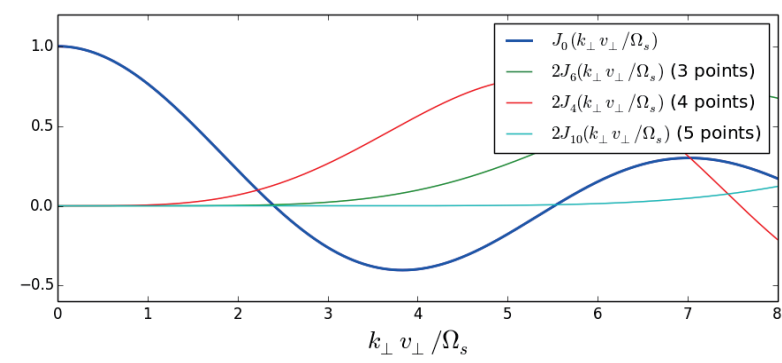

Fig. 2 Exact result of the simple averaging of a mode $k_{\perp}$ (blue) and the error terms for various number of points.

what extent the number of points $M$ controls the error.

As stated in equation (8), the simple averaging is easily expressed in Fourier space for a mode $k_{\perp}$ as $\widehat{\langle\Phi\rangle}_{k}=$ $J_{0}\left(k_{\perp} v_{\perp} / \Omega_{\mathrm{s}}\right) \widehat{\Phi}_{k}$. Fortunately, the numerical value of our simple averaging is also easy to express this way:

$$
\begin{aligned}
\widehat{\langle\Phi\rangle_{k} / \widehat{\Phi}_{k}=} & J_{0}\left(\frac{k_{\perp} v_{\perp}}{\Omega_{\mathrm{s}}}\right) \\
& +J_{M}\left(\frac{k_{\perp} v_{\perp}}{\Omega_{\mathrm{s}}}\right)+J_{-M}\left(\frac{k_{\perp} v_{\perp}}{\Omega_{\mathrm{s}}}\right) \\
& +J_{2 M}\left(\frac{k_{\perp} v_{\perp}}{\Omega_{\mathrm{s}}}\right)+J_{-2 M}\left(\frac{k_{\perp} v_{\perp}}{\Omega_{\mathrm{s}}}\right) \\
& +\ldots
\end{aligned}
$$

Hence, using the fact that $J_{M}=(-1)^{M} J_{-M}$, the first error term will be either $2 J_{M}\left(k_{\perp} v_{\perp} / \Omega_{\mathrm{s}}\right)$ for $M$ even or $2 J_{2 M}\left(k_{\perp} v_{\perp} / \Omega_{\mathrm{S}}\right)$ for $M$ odd. In a numerical simulation where $k_{\perp} v_{\perp} / \Omega_{\mathrm{S}}$ is finite, not so many points are necessary to keep this error close to 0 (cf. Fig. 2) especially if it is an odd number. As all variables in the field solver are integrated over $v_{\perp}$, the high relative error in one of the the narrow intervals around $J_{0}$ 's zeros is never an issue in practice.

\section{Numerical Resolution of the GK Quasi-Neutrality Condition}

Once the matrices for the simple and double gyroaveragings have been computed using the methods presented above, the GK quasi-neutrality condition (5), can be written in a matricial form (assuming $T_{\mathrm{e}}=T_{\mathrm{i}}$ and $e_{\mathrm{i}}=e$ for simplicity) as $2 \Phi-A \Phi=C$ where $A$ is the matrix for the double averaging operator and $C$ includes the RHS (the flux averaging term has been dropped temporarily). Although the GK quasi-neutrality condition is only a 3dimensional equation, solving this equation numerically can be a heavy task as the linear system is quite large $\left(N_{\varphi}\right.$ systems of size $N_{r} \cdot N_{\theta}$ or $N_{R} \cdot N_{Z}$, with $N_{\varphi}$ the number of mesh points in the $\phi$ direction etc) and the large diameter and complexity of the orbits used in the simple and double averagings reduce the sparsity of the matrices. Therefore, instead of usual linear solving methods (LU decomposition, Cholesky decomposition, etc), a specificity of the equation is used to solve it through a fix point method us- ing the form:

$$
C+(A-1) \Phi=\Phi .
$$

Starting from an initial guess of the solution $\Phi_{0}$, the relation $\Phi_{n+1}=C+(A-1) \Phi_{n}$ will converge towards a solution if and only if $A-1$ is a contractor (the absolute value of all eigenvalues are strictly smaller than 1 ). Fortunately, as can be seen in equation (9), the Fourier transform in Cartesian coordinates diagonalises the double averaging operator, making its eigenvalues explicit, the eigenvalues of $A-1$ are $1-\Gamma_{0}\left(k_{\perp}^{2}\right) \in[0,1]$. If this confirms the converge of the fixed point method, each iteration only reduces the distance to the solution by a factor $1-\Gamma_{0}\left(k_{\perp \text {,max }}^{2}\right)$ which is likely to be close to 1 , making the convergence potentially very slow under this form. Adding $(\alpha-1) \Phi$ on each side of the equation we obtain the form

$$
\frac{1}{\alpha}\{C+[A+(\alpha-2)] \Phi\}=\Phi,
$$

for which $\alpha$ can be used as a control parameter to improve the convergence speed of the method. The optimal value of this parameter can be easily calculated as

$$
\alpha=\frac{\|A-2\|^{2}}{\langle A-2,1\rangle} .
$$

When reintroducing the magnetic flux surface average term that was temporarily dropped, the additional term that is evacuated by first solving the flux averaged version of this equation, the so called Zonal Flow (ZF) equation:

$$
\overline{2 \Phi-\langle\Phi \Phi\rangle-\bar{\Phi}}=\bar{C} \quad \text { i.e. } \quad\left(1-A_{\mathrm{ZF}}\right) \bar{\Phi}=\bar{C} .
$$

The coefficients of the flux averaged double averaging matrix $A_{\mathrm{ZF}}$ are computed from the coefficients of the double averaging through a projection on an arbitrary radial line of the sampling points along the flux surfaces and the system is solved by a simple LU method, being small enough (1D) to allow such a simple method. If the flux averaging of $C$ and set up of this equation are simple in polar coordinates for a simple circular configuration, the computation is much harder in the general case, but the computation time always remains small compared to the rest of the code.

In terms of complexity, the fix point method usually converges in about 6 iterations, independently of the mesh numbers or physical parameters. The longest part of one iteration is the computation of the double gyro-averaging is equivalent to very optimised sparse matrix multiplication, making the total complexity of the order of $O\left(N_{\perp}^{2}\right)$ (with $N_{\perp}$ the number of point in a poloidal plane), compared to the $O\left(N_{\perp}^{3}\right)$ of an LU resolution for instance. The computation in real space of the double averaging is also parallelised more efficiently than the matricial operations involved in an LU resolution of a linear system making the overall use of the fixed point method very advantageous. Overall the parallelisation of the code is close to the ideal case. 

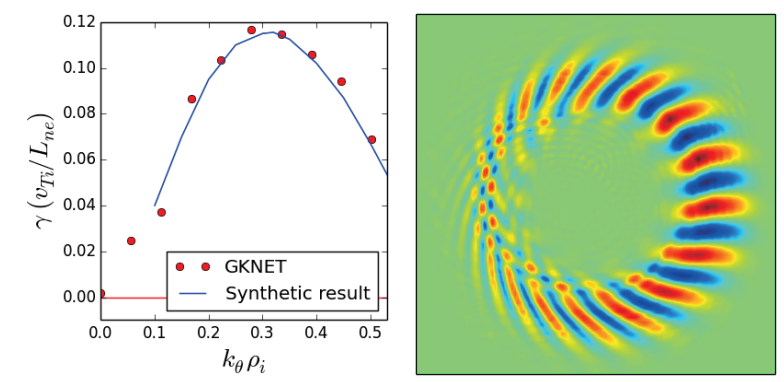

Fig. 3 Linear mode growth rates versus $k_{\theta} \rho_{i}$ for Cycle DIII-D system compared with a synthetic result as found in [5] (left) and poloidal cross section of the electric potential's most unstable toroidal mode (right).

\section{Numerical Results}

To verify the accuracy of the code, it was benchmarked using two standard tests for gyrokinetic simulation codes: the DIII-D base case $[4,5]$ as well as a collisionless zonal flow damping test.

The reference dimensionless parameters for the DIIID base case are: $R_{0} / L_{T}=6.92, R_{0} / L_{n}=2.2, r_{p} / R_{0}=0.18$, $q=1.4, s \equiv(r / q)(\mathrm{d} r / \mathrm{d} q)=0.8$ and $T_{\mathrm{e}} / T_{\mathrm{i}}=1$. Additionally, the domain in velocity space was restricted to $-5 v_{T \mathrm{i}} \leq v_{/ /} \leq 5 v_{T \mathrm{i}}$ and $0 \leq \mu \leq 12.5 B_{0} / T_{\mathrm{e}}$; the profile $q(r)=0.85+2.18(r / a)^{2}$ was used so that $q\left(r_{p}\right)=1.4$ and $\hat{s}\left(r_{p}\right)=0.8$. Boundary conditions were set to 0 outside of the domain. The simulation was performed on a grid $\left(N_{R}, N_{Z}, N_{\varphi}, N_{v_{l}}, N_{\mu}\right)=(64,64,128,32,16)$ using 9 points per circle and third order interpolations in the field solver. The magnetic flux surface term in eq. (5) is ignored for this type of simulations. As shown on Fig. 3, our numerical results are in good agreements with results obtained by other codes.

In the second benchmark test, an initial perturbation of the form $\delta f=10^{-3} \sin \left(\frac{\pi}{2}(1-r / a)\right)$ leading to the collisionless damping of Geodesic Acoustic Modes (GAMs). Most parameters are the same as in the previous case, except that $R_{0} / L_{t}=\infty, R_{0} / L_{n}=\infty$ (i.e. $T=1$ and $n_{0}=1$ ) and $q(r)=1.5+1.5(r / a)^{2}$ leading to $q\left(r_{p}\right)=1.9$. The expected residual value of the zonal flow as well as the

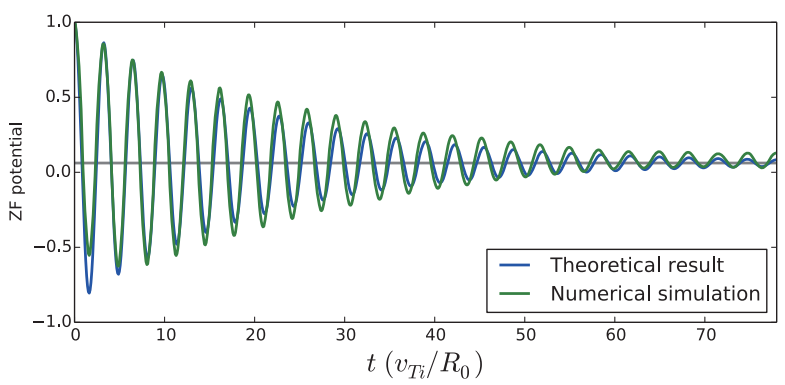

Fig. 4 Time evolution of the zonal flow potential compared with the theoretical result as given in $[6,7]$.

damping rate have been theoretically calculated $[6,7]$ and are plotted alongside our numerical result on Fig. 4 showing a very good agreement.

\section{Conclusion}

A new 5D full-f GK Vlasov code, GKNET, has been developed, using real space computation of the gyrokinetic operators. It operates under very general assumptions, allowing proper treatment of finite Larmor radius effects and any magnetic configuration. Benchmarks test validate the accuracy of the code in good agreements with the results found in the literature. In the future, this code will be used in realistic magnetic configurations such as Dshape, X-point and negative D-shape. We will also implement kinetic electrons to study ITG+TEM nodes in global system, in which our rigorous treatment of FLR effects may be essential.

[1] Z. Lin and W.W. Lee, Phys. Rev. E 52, 5646 (1995).

[2] Y. Idomura et al., Comput. Phys. Commun. 179, 391 (2008).

[3] G. Dif-Pradalier et al., Commun. Nonlinear Sci. Numer. Simul. 13, 65 (2007).

[4] V. Grandgirard et al., Commun. Nonlinear Sci. Numer. Simul. 13, 81 (2008).

[5] A.M. Dimits et al., Phys. Plasmas 7, 969 (2000).

[6] M.N. Rosenbluth and F.L. Hinton, Phys. Rev. Lett. 80, 724 (1998).

[7] H. Sugama and T.-H. Watanabe, Phys. Plasmas 13, 012501 (2006). 